\title{
Probabilistically Bounded Soft Sphere Detection for MIMO-OFDM Receivers: Algorithm and System Architecture
}

\author{
Predrag Radosavljevic, Member, IEEE, Yuanbin Guo, Member, IEEE, and \\ Joseph R. Cavallaro, Senior Member, IEEE
}

\begin{abstract}
Iterative soft detection and channel decoding for MIMO OFDM downlink receivers is studied in this work. Proposed inner soft sphere detection employs a variable upper bound for number of candidates per transmit antenna and utilizes the breath-first candidate-search algorithm. Upper bounds are based on probability distribution of the number of candidates found inside the spherical region formed around the received symbolvector. Detection accuracy of unbounded breadth-first candidatesearch is preserved while significant reduction of the search latency and area cost is achieved. This probabilistically bounded candidate-search algorithm improves error-rate performance of non-probabilistically bounded soft sphere detection algorithms, while providing smaller detection latency with same hardware resources. Prototype architecture of soft sphere detector is synthesized on Xilinx FPGA and for an ASIC design. Using area-cost of a single soft sphere detector, a level of processing parallelism required to achieve targeted high data rates for future wireless systems (for example, 1 Gbps data rate) is determined.
\end{abstract}

Index Terms-Sphere detection, iterative detection-decoding, hardware implementation, FPGA prototype, ASIC synthesis, Gbps data throughput.

\section{INTRODUCTION}

$\mathbf{F}$ UTURE wireless receivers are targeted to support hundreds of MBits/sec data-rates with excellent quality of service. In addition, high and flexible spectral efficiency is desired. In order to achieve this, it is proposed that multiple transmit antennas are accompanied with multiple receive antennas forming multiple-input multiple-output (MIMO) wireless transceivers [1]. Orthogonal Frequency Division Multiplexing (OFDM) transmission scheme [2] has been widely accepted for future wireless standards. It transforms highly scattering channel environments into frequency non-selective sub-carrier channels that are equalized with decent computational complexity. However, a major challenge nowadays is to design high performance MIMO-OFDM receiver that efficiently mitigates strong interference from multiple transmit antennas. Minimum mean squared error (MMSE) equalizers interfaced with soft-input soft-output (SISO) decoders are proposed in [3], [4]. The a posteriori probabilities (APPs)

Manuscript received 30 October 2008; revised 28 May 2009.

P. Radosavljevic is with Patterson\&Sheridan, LLP, Houston, TX 77056 USA (e-mail: pradosavljevic@pattersonsheridan.com).

Y. Guo is with Huawei, San Diego, CA 92121 USA (e-mail: yuanbinguo@huawei.com).

J. R. Cavallaro is with the Department of Electrical and Computer Engineering, Rice University, Houston, TX 77005 USA (e-mail: cavallar@rice.edu).

Digital Object Identifier 10.1109/JSAC.2009.091002. of transmitted coded bits are iteratively improved between outer decoder and inner equalizer. However, in systems with high spectral efficiency, such as the system with four transmit/receive antennas and 16-QAM modulation, the error-rate performance is still far away from the channel capacity. To approach the channel capacity, authors in [5] propose the soft sphere detection (SSD) as an approximation of exponentially complex maximum a posteriori (MAP) detection. However, computational complexity of this scheme is prohibitively high. Sub-optimal SSD algorithms have been also recently proposed in [6], [7], [8]. However, the major drawback of these solutions is either significant error-rate performance degradation [7], [8], or a large latency of the candidate-search algorithm [6].

In this paper, we propose a novel soft sphere detection approach with bounded candidate-search based on a probabilistic model. Search bounds are based on probability distributions for the number of candidates found inside the hyper-sphere in different search levels. Significant reduction of area cost and search latency is achieved compared to the search with no bounds but with the same sphere radius, while detection accuracy is preserved. The proposed soft sphere detection with variable bound per search level is suitable for achieving high data rates required by emerging wireless standards, such as data rates in the order of 1 Gbps. The cost-efficient high-throughput architecture design of soft sphere detector is proposed that can be iteratively interfaced with outer soft-input soft-output (SISO) maximum a posteriori (MAP) decoder.

The paper is organized as follows. Section II introduces MIMO wireless system model, soft sphere detection and iterative detection/decoding. The bounded soft sphere detection (BSSD) algorithm is proposed in Section III. Highlevel architecture design of BSSD is described in Section IV. Resource utilization of FPGA prototype and ASIC design of a single soft sphere detector are given in Section V. The processing parallelism of iterative detector-decoder required to meet high throughput requirements for future wireless systems (such as 1 Gbps data rate) is estimated in Section VI. The paper is concluded in Section VII.

\section{MiMO Wireless System AND SPHeRe Detection}

The coded MIMO OFDM wireless system with iterative detection and decoding at the receiver is shown in Fig. 1. It is assumed a system model with 256 subcarriers, which is compatible with the 3GPP-LTE wireless standard [9]. All 


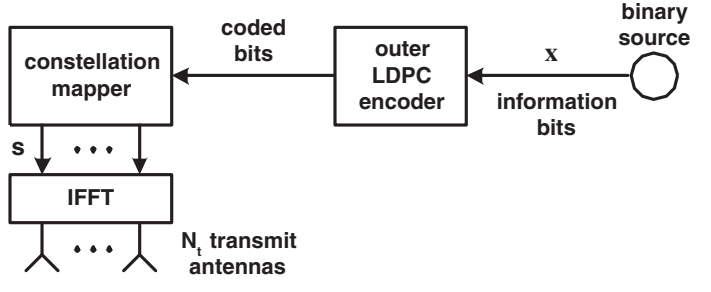

$\mathrm{H}$

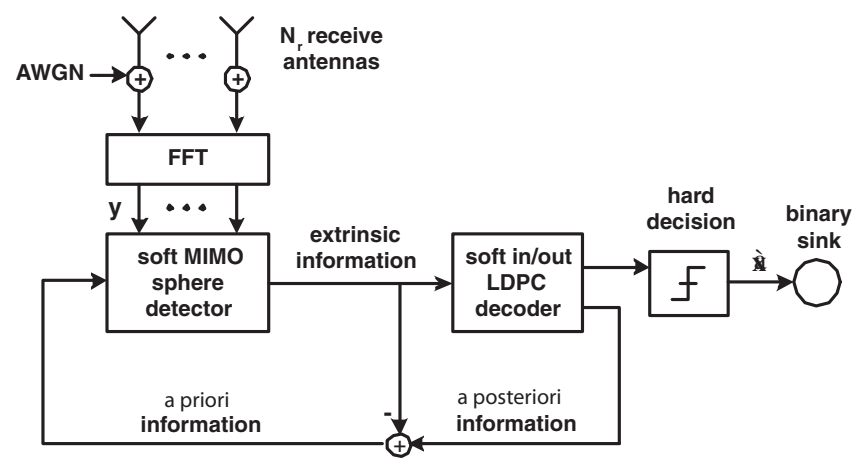

Fig. 1. MIMO transceiver, iterative detection and decoding at the receiver.

subcarriers are utilized as data subcarriers, while no pilot tones are considered in simulations since perfect channel knowledge is assumed at the receiver side. Symbols (i.e., constellation points) that are simultaneously transmitted from all antennas are mapped to the same subcarrier position. This system can be defined by the linear equation:

$$
\mathbf{y}=\mathbf{H s}+\mathbf{n},
$$

where $\mathbf{y}$ is a vector of $N_{r}$ received symbols, $\mathbf{H}$ is the $N_{r}$ $\times N_{t}$ matrix of flat-fading channel coefficients per subcarrier channel, $\mathbf{s}$ is a vector of $N_{t}$ transmitted modulated symbols, $\mathbf{n}$ is additive noise at the receiver, and $N_{t}$ and $N_{r}$ are the number of transmit and receive antennas, respectively. The size of both inverse fast Fourier transform (IFFT) and fast Fourier transform (FFT) shown in Fig. 1 is 256 in accordance with the number of considered subcarriers. The IFFT and FFT blocks illustrated in Fig. 1 are common for all transmit and receive antennas, respectively.

The Maximum Likelihood (ML) detection of the transmitted symbols from the candidate set $\Lambda$ is given by:

$$
\hat{\mathbf{s}}_{M L}=\arg \min _{\mathbf{s} \in \Lambda}\|\mathbf{H s}-\mathbf{y}\|^{2} .
$$

Since the number of possible transmitted bit-vectors per channel realization is $2^{N_{t} \cdot M_{C}}$ (where $M_{C}$ is the number of bits per transmitted constellation symbol), the ML approach is not practical for cost-efficient hardware implementation of receivers with high spectral efficiency. On the other hand, sphere detection [10] represents a simplification of the ML detection where tested candidates are constrained to those that lie inside the hyper-sphere with radius $r$ around initial solution based on received symbol-vector $\mathbf{y}$ :

$$
P(\mathbf{s})=\|\mathbf{H s}-\mathbf{y}\|^{2} \leq r^{2} .
$$

In order to reduce computational complexity, (3) is transformed after applying the $\mathrm{QR}$ decomposition $(\mathbf{H}=\mathbf{Q R})$ into an identical recursive problem. The matrix $\mathbf{R}$ is $N_{t} \times N_{t}$ upper triangular, and matrix $\mathbf{Q}$ is unitary $N_{r} \times N_{t}$ matrix. Equation 3 now becomes:

$$
P(\mathbf{s})=\left\|\mathbf{R s}-\mathbf{Q}^{H} \mathbf{y}\right\|^{2} \leq r^{2} .
$$

Since matrix $\mathbf{R}$ is upper triangular, the Euclidian distance $P(\mathbf{s})$ can be calculated recursively from one transmit antenna to another:

$P_{m}(\mathbf{s})=P_{m+1}(\mathbf{s})+\left|\hat{y}_{m}-\sum_{j=m}^{N_{t}} R_{m j} s_{j}\right|^{2} \leq r^{2}, m=N_{t}, \ldots, 1$,

where $\hat{\mathbf{y}}=\mathbf{Q}^{H} \cdot \mathbf{y}$ is the center of the hyper-sphere, and $P_{N_{t}+1}(\mathbf{s})=0$ for all possible transmitted vectors $\mathbf{s} \in \Lambda$. The term $P_{m}(\mathbf{s})$ represents partial Euclidian distance (PED) from the center of hyper-sphere to the partial vector-candidate $\mathbf{s}_{m}$. It is computed as a cumulative distance for candidates of transmit antennas $N_{t}, N_{t}-1, \ldots, m . R_{m j}$ represents entry of matrix $\mathbf{R}$ from the $m$-th row and the $j$-th column. This algorithm can be viewed as a tree-traversal with the root at level $m=N_{t}+1$. Nodes of the tree at the $m$-th search level correspond to the constellation points that are inside the sphere with the chosen radius $r$ for the $\left(N_{t}-m+1\right)$-th transmit antenna. Nodes outside the sphere and the corresponding subbranches are discarded from the search process.

Soft sphere detection (SSD) algorithm represents an extension of the original sphere detection approach. Instead of finding only the ML candidate, the SSD scheme finds a list of candidates $\mathcal{L}$. Based on this list of candidates, the reliability information for each transmitted bit $x_{k}$ is computed and passed as an extrinsic probability to outer maximum a posteriori (MAP) decoder for further improvement of reliability. The extrinsic probabilities at the input of MAP decoder can be computed as in [5] using the max-log approximation:

$$
\begin{array}{r}
L_{E}\left(x_{k} \mid y\right) \quad \approx \frac{1}{2} \max _{\mathbf{x} \in \mathcal{L} \cap \mathbb{X}_{k,+1}}\left\{-\frac{1}{\sigma^{2}}\|\mathbf{y}-\mathbf{H} \cdot \mathbf{s}\|^{2}+\mathbf{x}_{[k]}^{T} \cdot \mathbf{L}_{A,[k]}\right\} \\
-\frac{1}{2} \max _{\mathbf{x} \in \mathcal{L} \cap \mathbb{X}_{k,-1}}\left\{-\frac{1}{\sigma^{2}}\|\mathbf{y}-\mathbf{H} \cdot \mathbf{s}\|^{2}+\mathbf{x}_{[k]}^{T} \cdot \mathbf{L}_{A,[k]}\right\},(6)
\end{array}
$$

where $\mathbf{x}_{[k]}$ is the sub-vector of $\mathbf{x}$ obtained by omitting the $k$-th bit $x_{k}, \mathbf{L}_{A,[k]}$ is the vector of all a priori probabilities $L_{A}$ for transmitted vector $\mathbf{x}$ obtained by omitting $L_{A}\left(x_{k}\right), \sigma^{2}$ is the noise variance, $\mathbb{X}_{k,+1}$ is the set of $2^{N_{t} \cdot M_{C}-1}$ bits of vector $\mathbf{x}$ with $x_{k}=+1$, while $\mathbb{X}_{k,-1}$ is similarly defined.

\section{Bounded Soft Sphere Detection}

A soft sphere detection with variable search bounds per search level is proposed in this work. The candidate-search process is based on a breadth-first principle: all valid candidates for one transmit antenna are found before starting a search process for the next transmit antenna based on previously found candidates. The breadth-first K-best scheme from [11], [8] assumes that at the end of every search level the best $K$ candidates are preserved as parent-nodes in the subsequent search level. The K-best detection approach is suitable for pipelining because of a constant processing latency per search level, and it can achieve a high detection throughput. On the other hand, the K-best approach suffers error-rate 
performance loss because a large number of candidates are discarded at every search level. A similar QRD-M detection algorithm from [7] assumes that after every search level the best $M$ search-branches are preserved. At the beginning, transmit antennas are reordered based on channel gain from each antenna. There is no notion of the sphere or the sphere radius value in both $\mathrm{K}$-best and QRD-M detection algorithms, but only limited number of candidates closest to the received point are considered at every search level of the candidatesearch process. Authors in [6] propose design of soft sphere detection where the depth-first search with maximum of 256 search operations is employed. One search operation is defined as a simultaneous testing of all constellation points if they are inside the hyper-sphere based on the cumulative distance from previous search levels, as illustrated in (5). The depth-first search assumes that every next search operation is performed in a different search level such as in [12]. The bounded depthfirst search from [6] achieves a decent error-rate performance but the search process is long because of the large number of search operations.

\section{A. Probability based search process}

In order to preserve detection accuracy and to decrease the probability of discarding good candidates in early search levels including the ML candidate, a detection algorithm is proposed where the search bounds are based on distribution of the number of candidates found inside the sphere for different search-levels. The search bounds are variable per search-level unlike in the K-best and QRD-M algorithms, and sorting of candidates is not employed. The search process is stopped once the pre-determined maximum number of valid candidates $M_{a x}$ for the $l$-th search-level is reached, and continues in the next $(l+1)$-th search level based on previously found candidates. Candidates that exceed the predetermined bound are discarded from the search process. The number of search operations is upper-bounded by $1+\sum_{l=1}^{N_{t}-1} \mathrm{Max}_{l}$, which determines the largest latency of the search process. This approach is named a bounded soft sphere detection (BSSD), and Fig. 2 illustrates the structure of the search-tree.

Search-bounds are determined in such a way to preserve detection accuracy of the breadth-first search algorithm with no bounds per search level, while the sphere radius is the same. The goal is also to reduce memory space for storing partial/final candidates and Euclidean distances during the search process, and to substantially decrease latency of the search process compared to the unbounded breadth-first search. The pre-determined search-bound for particular searchlevel is based on probability that a certain number of candidates are found inside the hyper-sphere considering a large number of channel realizations. Figure 3 shows distributions of the number of valid candidates for different search-levels if $\operatorname{Max}_{2}=\operatorname{Max}_{3}=100$. Frequency non-selective Rayleigh fading channels per subcarriers can be considered, while the system employs four transmit/receive antennas and 16-QAM modulation. Total constellation power is normalized in order to insure unitary transmit energy. The sphere radius is fixed providing on average about 25 final candidates.

At the beginning of the search process, majority of tested candidates are inside the sphere: PEDs are small and candi-

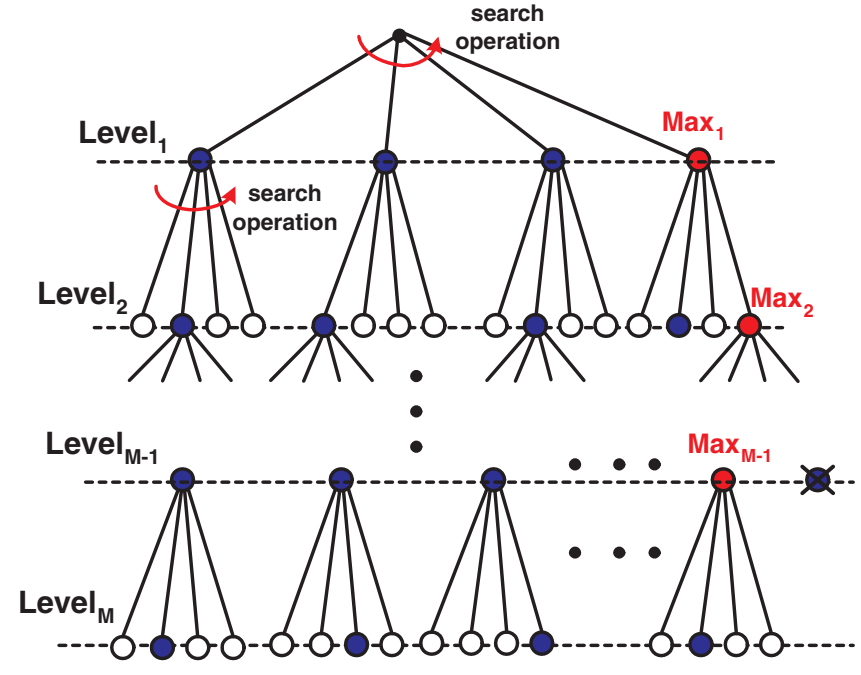

Fig. 2. The search-tree visualization of bounded search with variable bounds per search level: bounded soft sphere detection $\operatorname{BSSD}\left(\operatorname{Max}_{1}, \operatorname{Max}_{2}, \ldots, \operatorname{Max}_{N_{t}-1}\right) . \quad N_{t}$ transmit antennas, QPSK modulation is assumed.

dates are unreliable. This is the reason why the K-best solution from [8] and the QRD-M [13] suffer significant error-rate performance loss: the probability of discarding good candidates including the ML candidate is high even after sorting of PEDs. On the other hand, the PEDs monotonically increase from one search level to another and a higher percentage of candidates in the lower search levels are outside the hypersphere as shown in Fig. 3. The number of candidates in the first search level cannot be bounded without significant errorrate performance loss. On the other hand, the search process in lower search levels can be stopped once a certain number of candidates is reached, and detection accuracy can be still preserved. This is because in lower search-levels a smaller percentage of candidates will reach the search-bound than in the first search level. Furthermore, by bounding the search process, a smaller percentage of partial and final candidates is stored in the memory than during the unbounded breadthfirst search with the same radius. Therefore, required memory space for storing partial/final candidates and their Euclidean distances is substantially decreased.

According to Fig. 3, the probability of discarding valid candidates in the $l$-th search level can be represented as:

$$
\operatorname{Pr}\left(\# \operatorname{cand}_{l} \geq \operatorname{Max}_{l}\right) \leq \gamma_{l},
$$

where $\#$ cand $_{l}$ is the number of candidates inside the hypersphere for the $l$-th search level, and $\gamma_{l}$ is the probability threshold value for a particular level chosen sufficiently small to preserve accuracy of the candidate-search process. It can be observed that the value of $\gamma_{l}$ is directly correlated with the search bound $\operatorname{Max}_{l}$ : larger search-bound causes smaller threshold and vice-versa. Typically, $\gamma_{l} \leq 0.1\left(l=2, \ldots, N_{t}-1\right)$ for preserving the detection accuracy of unbounded breadthfirst search. Since no bound for the number of candidates is established in the first search level, $\gamma_{1}=0$. It can be also noticed from Fig. 3 that $\gamma_{l} \geq \gamma_{l-1}$. This is because candidates inside the hyper-sphere in the lower $l$-th search level are more reliable than the candidates from the previous upper search level $l-1$. 

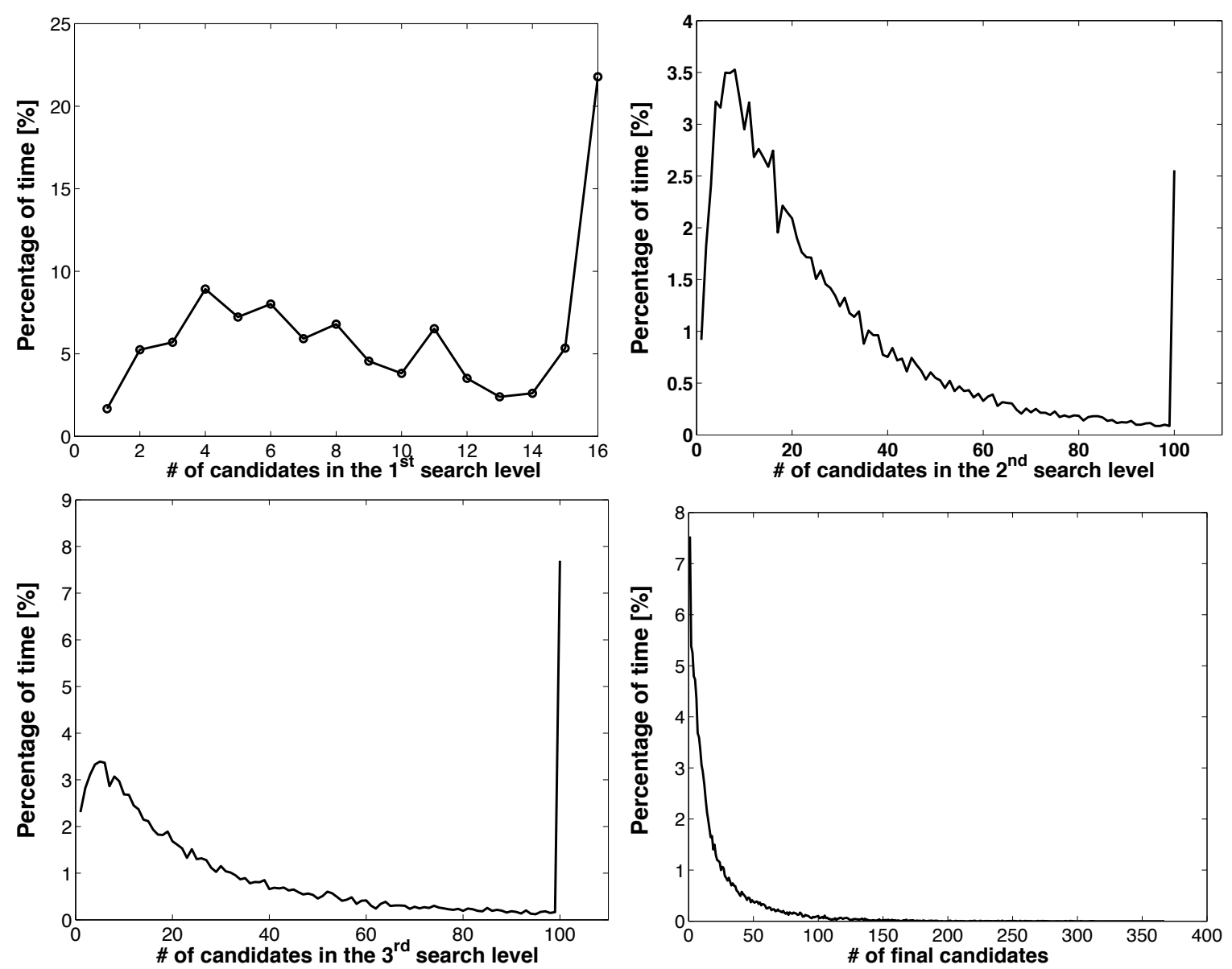

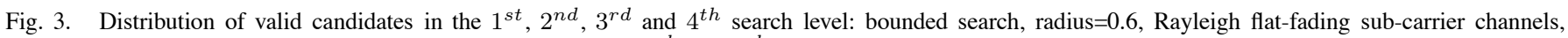
4x4 16-QAM. Maximum number of candidates is 100 in both $2^{\text {nd }}$ and $3^{r d}$ search levels. There is no sorting of transmit antennas.

Therefore, a larger fraction of the candidates can be discarded in lower search levels, and detection accuracy will remain the same.

It can be observed from Fig. 3 that chosen search bounds $\operatorname{Max}_{2}=\operatorname{Max}_{3}=100$ satisfy $\gamma_{l} \leq 0.1, l=2,3$ (i.e., $\gamma_{2} \approx 0.025$, $\gamma_{3} \approx 0.08$ ). It should be noted that these particular search bounds along with the chosen sphere radius of 0.6 represent one possible example of candidate search process that provide error-rate performance very close to the unbounded search algorithm, while search latency and implementation complexity is substantially reduced.

The previous analysis is conducted considering the 16QAM constellation size. In general, if the constellation size increases, transmitted modulated symbols are normalized with a larger factor in order to provide unitary transmit power. Because of that, a sphere radius should be chosen smaller because most of the candidates are located closer to the center of the sphere. Also, there is a larger number of candidates if the constellation size increases, and search bounds need to be larger in order to ensure that detection accuracy is not negatively affected. Although the sphere radius and the search bounds are fixed throughout this work, they can be also adjusted according to a signal-to-noise ratio (SNR) value at the receiver. If the SNR is higher, channel conditions are better and candidates that are located closer to the center of the sphere represent valid candidates. Therefore, the sphere radius and search bounds can be adjusted to be smaller. On the other hand, if the SNR is smaller, channel conditions are not worse and candidates are typically dispersed around the center of the sphere. Therefore, in order to ensure detection accuracy, the sphere radius can be adjusted to be larger and more candidates need to be evaluated.

1) BSSD with sorting of transmit antennas: It is proposed in this work to use the candidate-search process that starts with the most reliable transmit antenna - an antenna associated with the largest channel gain. Transmit antennas are ordered based on norms of columns of a sub-carrier channel matrix. This represents the H-norm ordering applied in [7]: transmit antenna with the largest channel gain corresponds to the largest column-norm. The distribution of candidates found inside the sphere when sorting of transmit antennas is applied are shown in Fig. 4 for four different search levels (i.e., for four transmit antennas).

Because of the sorting of transmit antennas, more reliable candidates are found at the beginning of the search process leading to more reliable final candidates. The identical system parameters are assumed as in the case when no sorting of transmit antennas is applied. After comparing Fig. 4 with Fig. 3, it can be observed that there is a more balanced distribution of candidates in the initial search level when sorting of transmit antennas is applied. Moreover, the probability distribution curves in lower search levels are shifted to the 

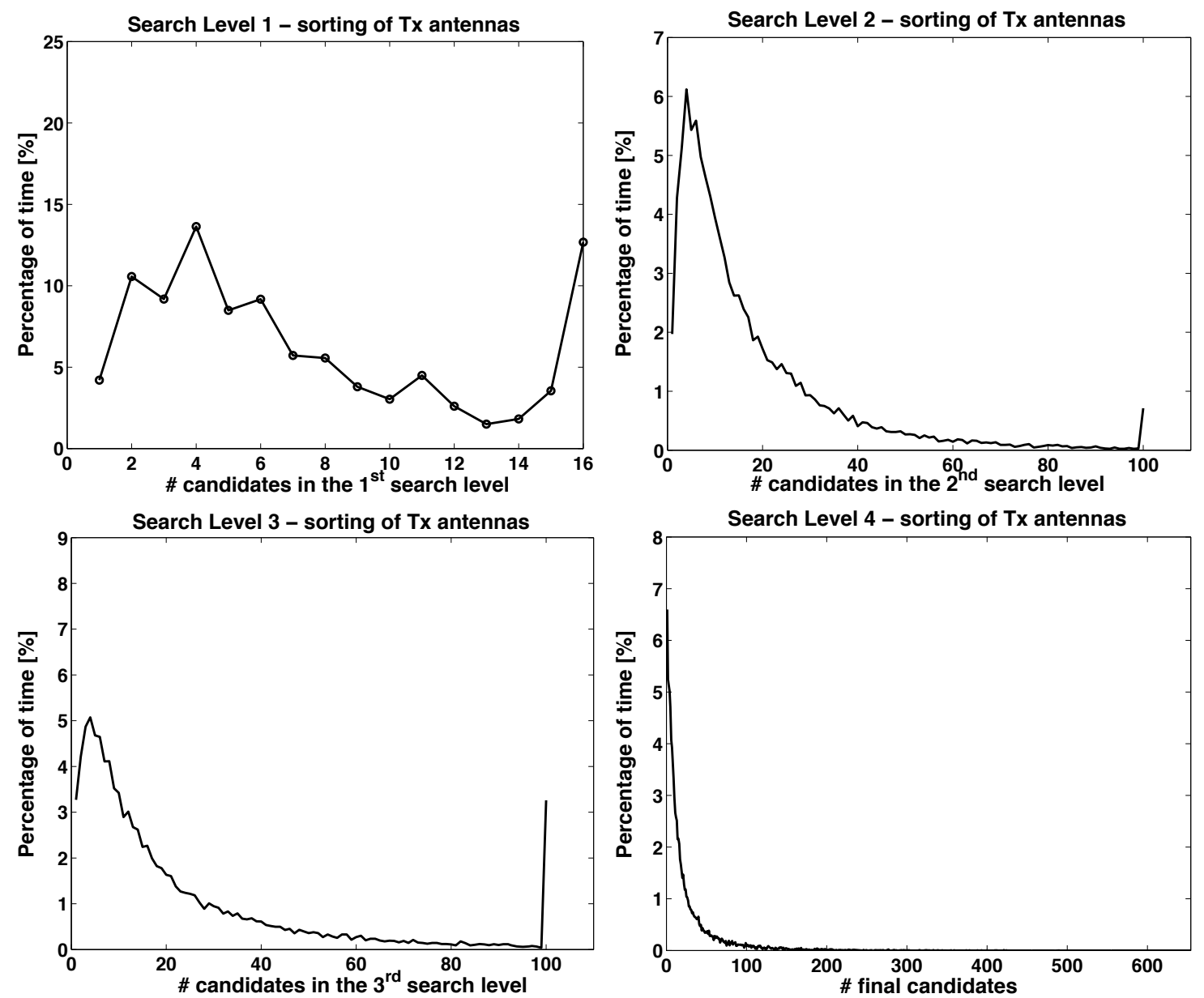

Fig. 4. Distribution of valid candidates in the $1^{\text {st }}, 2^{\text {nd }}, 3^{\text {rd }}$ and $4^{\text {th }}$ search level after sorting of transmit antennas based on the H-norm. Bounded breadth-first candidate-search, sphere radius $=0.6$, Rayleigh flat-fading sub-carrier channels, $4 \times 416-\mathrm{QAM}$. Maximum number of candidates is 100 in both $2^{\text {nd }}$ and $3^{\text {rd }}$ search levels.

left: the average number of candidates per search level is smaller. Although a smaller average number of candidates is found in every search level, these candidates are more reliable. Because of that the error-rate performance is improved and the average number of search operations is reduced.

Because the maximum number of valid candidates in the second and third search levels is bounded to some predetermined number, the probability distribution of valid candidates for these two search levels has a spike at the searchbound value (see Figures 3, 4). The search process stops when the pre-determined search-bound is reached.

2) Error-rate performance: Frame error rate (FER) performance for different detection schemes are shown in Fig. 5. It is assumed a wireless system with four transmit/receive antennas, 16-QAM modulation, Rayleigh frequency non-selective fading channel per sub-carrier, and a carrier frequency of $5 \mathrm{GHz}$. Outer decoding is based on low-density parity-check (LDPC) codes with 15 iterations of layered belief propagation, the codeword size is 1944 bits, the code rate is $1 / 2$, and there are up to four outer iterations between detector and decoder. The proposed detection approach is labeled as $\operatorname{BSSD}(16,100,100)$ specifying the maximum number of candidates in the first, second and third search levels, respectively. It can be observed from Fig. 5 that the error-rate performance of unbounded soft sphere detection (USSD) with the same sphere radius is preserved. Also, there is significant gain compared to the soft MMSE equalization with decision feedback (i.e., the MMSE-DFE scheme) from [3], and also compared to the soft QRD-M with $M=5$ final candidates (i.e., the Kbest breadth-first search) from [8]. The $\operatorname{BSSD}(16,100,100)$ has similar error-rate performance as the depth-first search from [6], while computational complexity is reduced thanks to better utilization of candidates from the initial search level: the proposed BSSD scheme with ordering of transmit antennas has 46 search operations on average versus the fixed number of 256 search operations of the algorithm from [6]. For the depth-first search, only a portion of valid candidates from the first search level is exploited as the parent-nodes in the lower search levels. Majority of search operations are executed in the final search level. Consequently, there is an implicit bounding of candidates from the first search level that are exploited in lower search levels. Our simulations in Rayleigh frequency non-selective sub-carrier channels show that approximately six candidates from the first search level are exploited in the depth-first search process, and approximately ten candidates on average in the proposed breadth-first $\operatorname{BSSD}(16,100,100)$ detection.

The computational complexity in terms of average number of search operations of different detection algorithms is shown in Fig. 6. If ordering of transmit antennas is ap- 


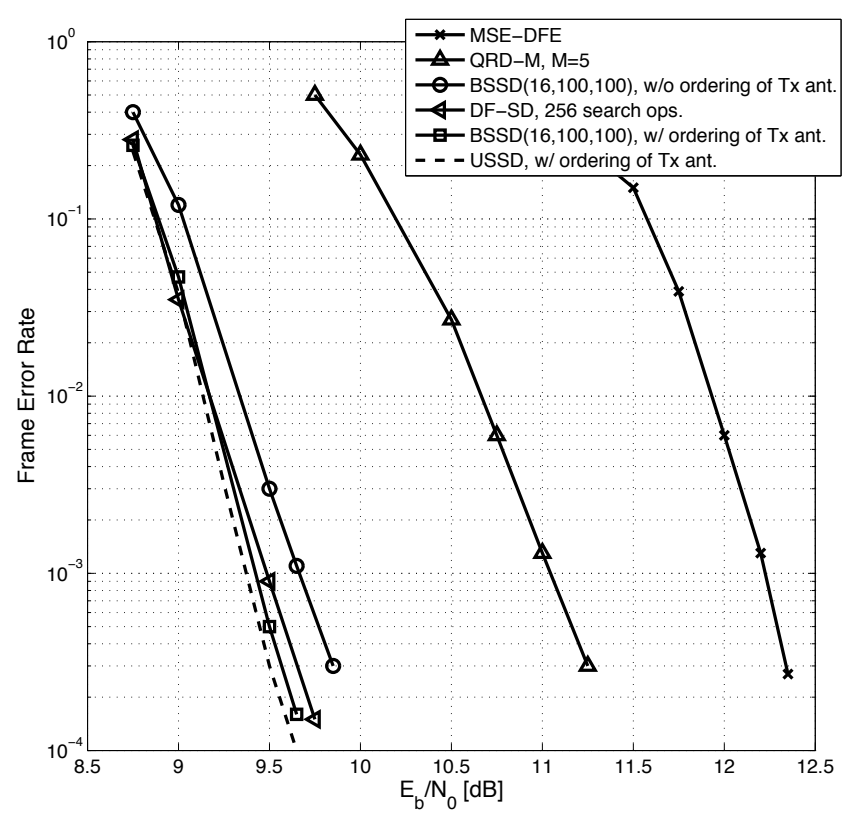

Fig. 5. Frame error rate performance of bounded soft sphere detection $\operatorname{BSSD}(16,100,100)$ vs. unbounded soft sphere detection (USSD) and other soft detection schemes for Rayleigh fading sub-carrier channels, 4x4 16-QAM. LDPC codeword size is 1944 bits, code rate is $1 / 2,15$ inner iterations of layered belief-propagation, up to four outer iterations between decoder and detector.

plied, then the average number of search operations for the $\operatorname{BSSD}(16,100,100)$ algorithm is decreased by approximately $25 \%$. The computational complexity of column-sorting (ordering of transmit antennas) is a minor part of the overall detection complexity, and it is beneficial both in terms of computational complexity and error-rate performance. It can be noted that the number of search operations is the smallest for the QRD-M detection since the small number of candidates are preserved after every search level (only $M=5$ candidates are preserved after every search level).

\section{ARCHITECTURE DESIGN OF BOUNDED SOFT SPHERE DETECTOR}

In this section, a high-level architecture design of the proposed bounded soft sphere detection (BSSD) is described. The sphere detector is prototyped on a Xilinx field programmable gate array (FPGA), and an application specific integrated circuit (ASIC) gate-count is estimated. A high level block diagram of the proposed soft sphere detector is shown in Fig. 7. There are several arithmetic blocks for computation of partial/final Euclidean distances (EDs) as well as for searching of candidates for each transmit antenna based on (5). Valid candidates for one transmit antenna (one search level) and their corresponding partial EDs are stored in the appropriate RAM block and used in the following search level. Once the final candidates are determined, they are used within the a posteriori probability (APP) function unit alongside the corresponding final ED. The APP FU computes the $a$ posteriori reliability information for coded bits based on the list of final candidates according to (6). Search for the list of final candidates is performed only during the initial iteration

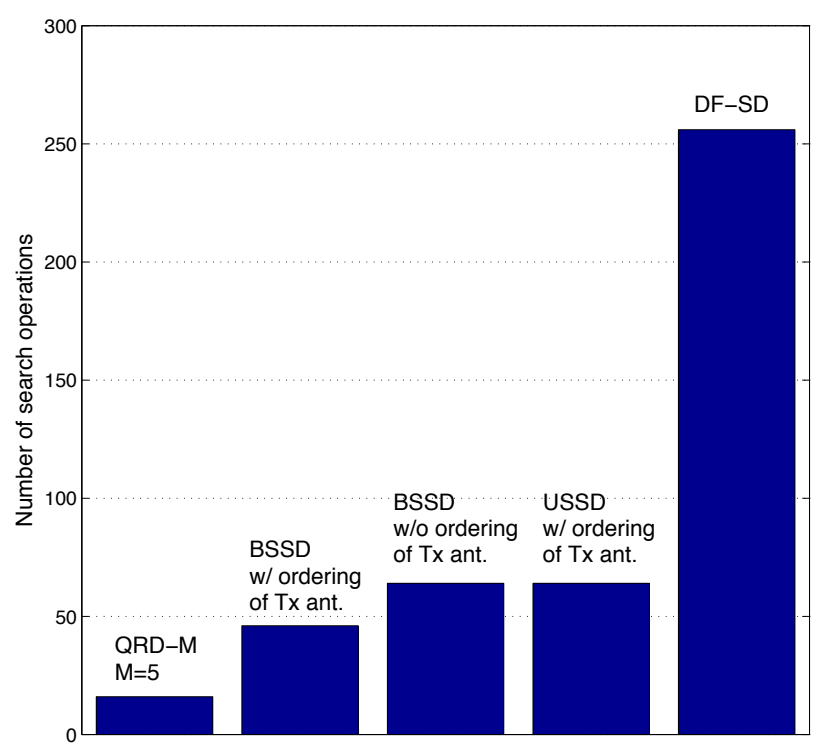

Fig. 6. Number of search operations for different detection algorithms in Rayleigh fading sub-carrier channels, 4 transmit/receive antennas, 16-QAM.

between inner detector and outer decoder. Outer feedback is employed between LDPC decoder and APP FU: improved $a$ posteriori information is based on improved a priori information from outer decoder and on the list of final candidates. Architecture implementation of the detector's sub-components are analyzed in more details.

\section{A. Arithmetic logic}

Arithmetic logic is composed of several sub-components as shown in Fig. 7: the pre-processing unit, candidate-search modules, and the APP function unit. Sorting of transmit antennas and QR decomposition of sub-carrier channel matrices are performed before the pre-processing stage, and their implementations are not analyzed in this work.

1) Pre-processing unit: The pre-processing unit calculates the center of the hyper-sphere, as well as the common factors defined in (9) used for testing all symbol-candidates for each transmit antenna. The center of the hyper-sphere is calculated as:

$$
\hat{\mathbf{y}}=Q^{H} \cdot \mathbf{y} .
$$

Factors $F_{m}$ are pre-computed in advance for all symbolcandidates according to:

$$
F_{m}=\hat{y}_{m}-R_{m m} \cdot s_{m}, \quad m=N_{t}, \ldots, 1 .
$$

Factors $F_{m}$ are computed for all $N_{t}$ different search levels (transmit antennas), saved in the registers, and utilized in the appropriate search-module from Fig. 7. However, it is not required to compute all $2 \cdot 2^{M_{C}}$ products $R_{m m} \cdot s_{m}$ per search level, where $M_{C}$ is the number of bits per constellation symbol: there are only eight different products in the case of 16QAM (i.e., four real and four imaginary parts) corresponding to the number of constellation levels. Furthermore, rather than computing products, it is possible to apply shift/add operations 


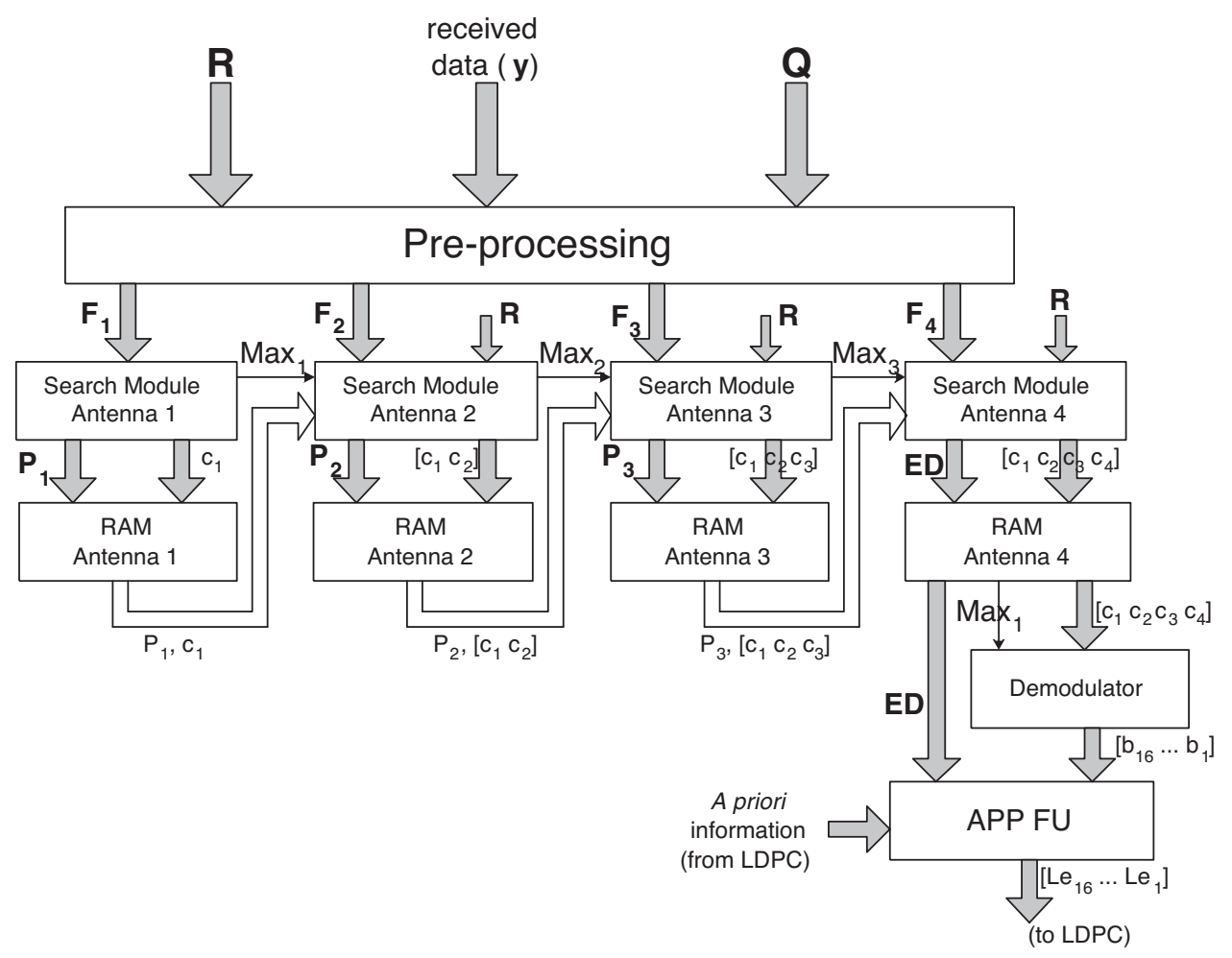

Fig. 7. Block-diagram of the soft sphere detector. Arithmetic logic (search for valid candidates in each search level) and RAM modules (storage of all valid candidates and their Euclidean distances after every search level).

on values $R_{m m}$ thanks to the known levels of constellation points.

2) Search modules: The search module simultaneously computes partial Euclidean distances (PEDs) for all $P_{C}=$ $2^{M_{C}}$ constellation points. All PEDs that are computed within a single search operation correspond to a common vectorcandidate found in the previous search level. Once computed, all PEDs are simultaneously tested if they are inside the sphere. Then, up to $P_{C}$ valid candidates along with their PEDs are saved in the memory for later use.

A wireless system with $N_{t}=4$ transmit antennas is assumed, and 16-QAM constellation size. Order in which transmit antennas are detected is irrelevant for the architecture design. The search-module for the first transmit antenna computes PEDs for all $2^{M_{C}}$ constellation points and checks if the constellation points are inside the pre-determined hyper-sphere according to:

$$
P_{1}^{q}=\left|F_{1}^{q}\right|^{2} \leq r^{2} ; \quad q=1, \ldots 2^{M_{C}} .
$$

For every valid candidate $c_{1}$ from the first search level, cumulative PEDs of the first and second transmit antennas are computed in the second search module according to:

$$
P_{2}^{q}=P_{1}\left(c_{1}\right)+\left|F_{2}^{q}-R_{12} c_{1}\right|^{2} \leq r^{2} ; \quad q=1, \ldots 2^{M_{C}} .
$$

For every valid partial vector-candidate $\left[c_{2} c_{1}\right]$ that corresponds to the second and first transmit antenna, cumulative PEDs for the first, second, and third transmit antennas are computed in the third search module. All partial candidates are tested if they are inside the hyper-sphere with radius $r$ according to:

$$
\begin{gathered}
P_{3}^{q}=P_{2}\left(c_{2}, c_{1}\right)+\left|F_{3}^{q}-R_{23} c_{2}-R_{13} c_{1}\right|^{2} \leq r^{2} ; \\
q=1, \ldots 2^{M_{C}} .
\end{gathered}
$$

Finally, in the last (fourth) search-module, the final EDs are computed and tested if they are inside the hyper-sphere for every valid vector-candidate $\left[c_{3} c_{2} c_{1}\right]$ corresponding to the third, second and first transmit antenna:

$$
\begin{gathered}
E D^{q}=P_{3}\left(c_{3}, c_{2}, c_{1}\right)+\left|F_{4}^{q}-R_{34} c_{3}-R_{24} c_{2}-R_{14} c_{1}\right|^{2} \\
E D^{q} \leq r^{2} ; \quad q=1, \ldots 2^{M_{C}}
\end{gathered}
$$

If the maximum pre-determined number of candidates for a particular search level is found, then the search process for that level stops. Final vector-candidates $\left[c_{4} c_{3} c_{2} c_{1}\right]$ are saved in the memory and used in the computation of APPs for the outer decoder.

For fully parallel computation of all products $R_{j m} \cdot c_{j}$ inside every search level $m\left(m=1, \ldots, N_{t}, j=1, \ldots, m-1\right)$, up to twelve $F U_{C S A}$ function units (check/shift/add function unit, see Fig. 8) and up to six add/subtract units are required. The $F U_{C S A}$ checks the constellation level of symbol-candidate $c_{j}$ for the $j$-th transmit antenna and then performs the appropriate shift, add/subtract operation and sign-conversion on real and imaginary parts of the $R_{j m}$ entry of upper triangular matrix R. Arithmetic logic used for computation of a single factor $R_{j m} \cdot c_{j}$ is shown in Fig. 8.

Computation of Euclidean distances from (13) can be rewritten as:

$$
E D^{q}=P_{3}\left(c_{3}, c_{2}, c_{1}\right)+|\operatorname{Re}\{X\}+i \operatorname{Im}\{X\}|^{2} \leq r^{2},
$$




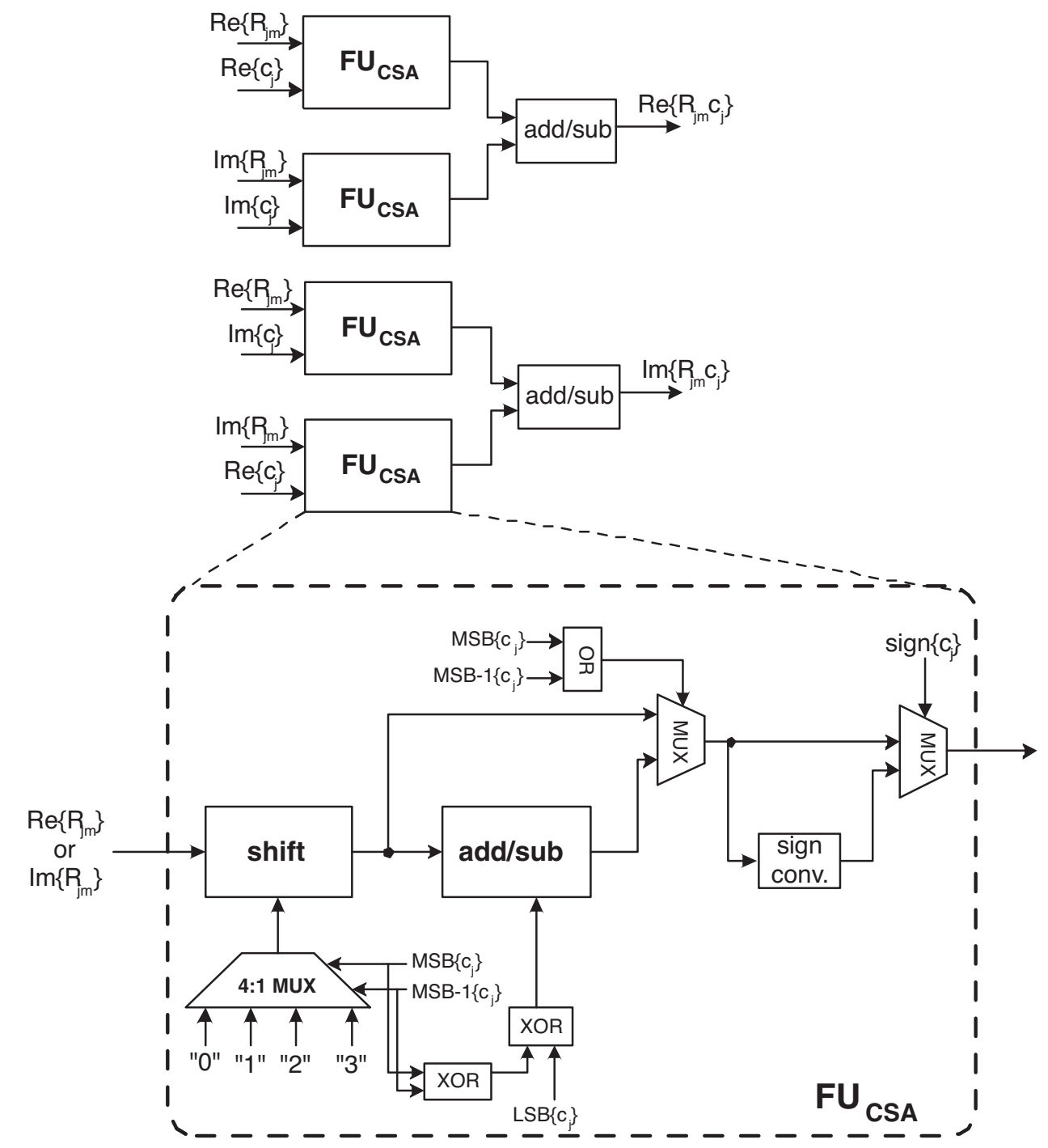

Fig. 8. Block diagram of arithmetic logic for computation of $R_{j m} \cdot c_{j}$ for a single value of $j$ in the case of 16-QAM modulation as a part of the search module for the $m^{t h}$ search level of the soft sphere detector from Fig. 7. Block diagram of the $F U_{C S A}$ for computation of partial $R_{j m} \cdot c_{j}$ products.

where $X=F_{4}-\sum_{j=1}^{3} R_{j 4} c_{j}$. It can be observed that there are only four different values of $\operatorname{Re}\{X\}$ and four different values of $\operatorname{Im}\{X\}$ in the case of 16-QAM. Therefore, fully parallel computation of $|X|^{2}$ requires eight square multipliers, and $P_{C}=2^{M_{C}}=16$ different values of $|X|^{2}$ are formed by the cross-addition of all individual results of square multiplications as is shown in Fig. 9.

\section{HARDWARE IMPLEMENTATION}

Implementation cost of an FPGA prototype for the proposed soft sphere detector is provided in this section, as well as the estimated hardware cost for the ASIC implementation. The fixed-point arithmetic precision is determined based on errorrate performance in different channel environments.

\section{A. Detection/decoding accuracy with fixed-point precision}

Figures 10, 11, and 12 show the frame error rate performance of iterative detection-decoding for floating and fixedpoint representation of numbers. An OFDM wireless system with four transmit/receive antennas and 16-QAM modulation is assumed. The following channel environments are respectively considered: the Rayleigh fading sub-carrier channel, a five-path indoor Winner A1-NLOS (no line of sight) channel with velocity of $5 \mathrm{~km} / \mathrm{h}$, and a two-path outdoor Winner B1NLOS channel with velocity of $35 \mathrm{~km} / \mathrm{h}$. Winner channel models [14] are extensively used to characterize wireless environments in an emerging $3^{\text {rd }}$ Generation Partnership Project Long Term Evolution (3GPP-LTE) standard [9]. It can be observed for 10-bit fixed-point arithmetic precision that there is a small performance loss of up to $0.25 \mathrm{~dB}$ in all channel environments. Therefore, this precision is chosen for computation and representation of Euclidean distances, as well as for representation and updating of reliability messages in the outer LDPC decoding algorithm.

\section{B. FPGA prototype cost}

A prototype architecture of the proposed bounded soft sphere detector $\operatorname{BSSD}(16,100,100)$ has been implemented in VHDL and synthesized for a Xilinx Virtex2P70 FPGA. 10-bit fixed-point arithmetic precision is used in the candidate-search 


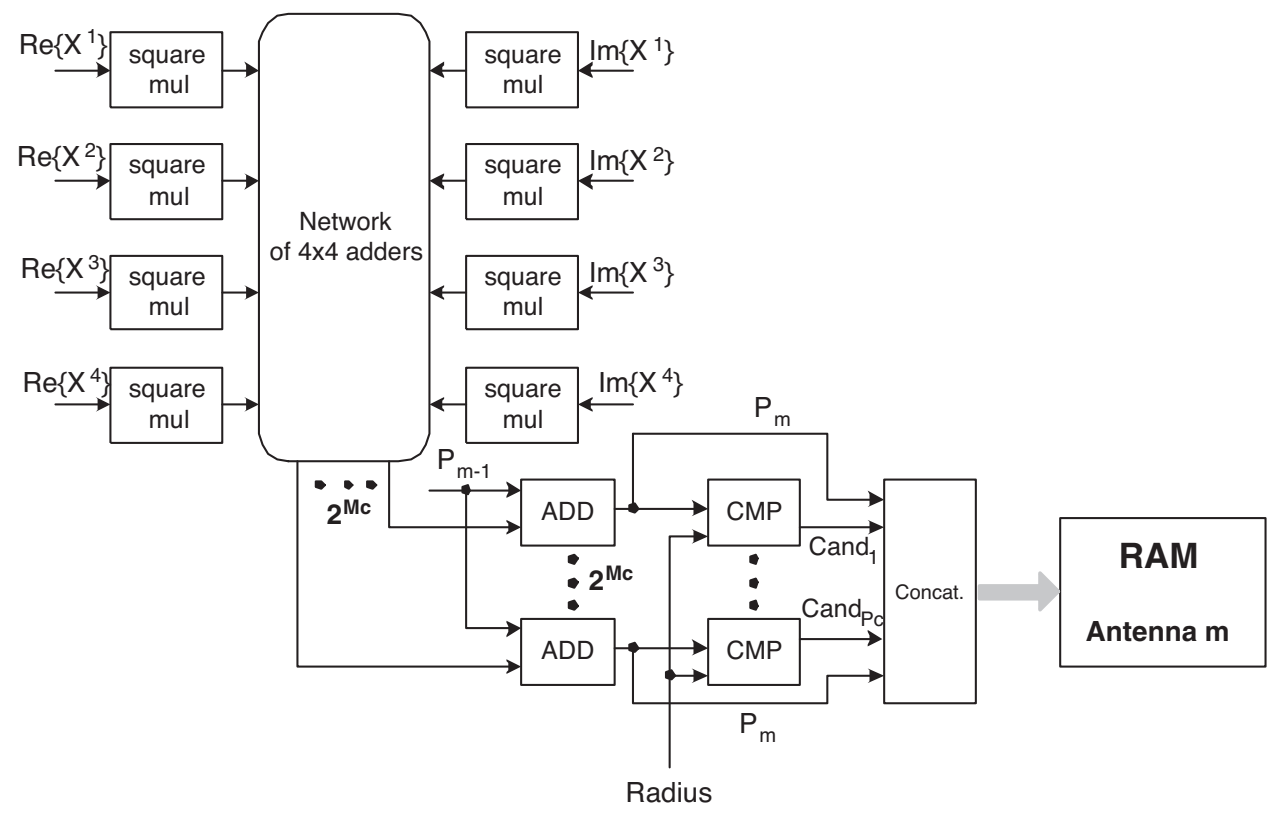

Fig. 9. Final stage of the search module for each transmit antenna $m, m=1, \ldots, N_{t}$ : parallel square operations, computation of the PEDs $P_{m}$ for all found candidates by cross-addition and comparison with the pre-determined sphere radius, $X=F_{m}-\sum_{j=1}^{m-1} R_{j m} c_{j}$.

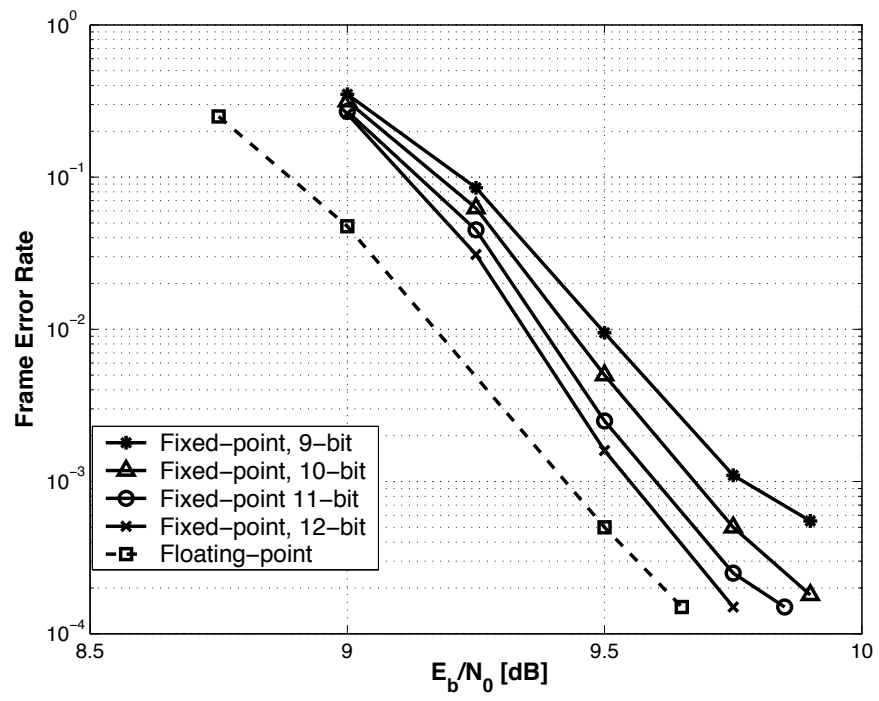

Fig. 10. Fixed vs. floating-point frame error rate performance of $\operatorname{BSSD}(16,100,100)$ detection; 4x4 16-QAM, Rayleigh sub-carrier fading channels. 10-bit arithmetic precision is chosen for implementation of BSSD algorithm and LDPC decoding.

algorithm, as well as for the representation of partial/final Euclidean distances. A wireless system with four transmit/receive antennas and 16-QAM modulation is assumed. Table I shows the FPGA utilization statistics of the prototype detector architecture based on the high-level design described in Section IV-A. Block-RAMs are used for storage of partial/final candidates and corresponding Euclidean distances during the search process. According to the Xilinx XST synthesis post place-and-route report, the maximum operational clock frequency is $49.50 \mathrm{MHz}$. This corresponds to the minimum data throughput of $1.82 \mathrm{MBits} / \mathrm{sec}$, while the average data throughput is approximately $8.60 \mathrm{MBits} / \mathrm{sec}$.

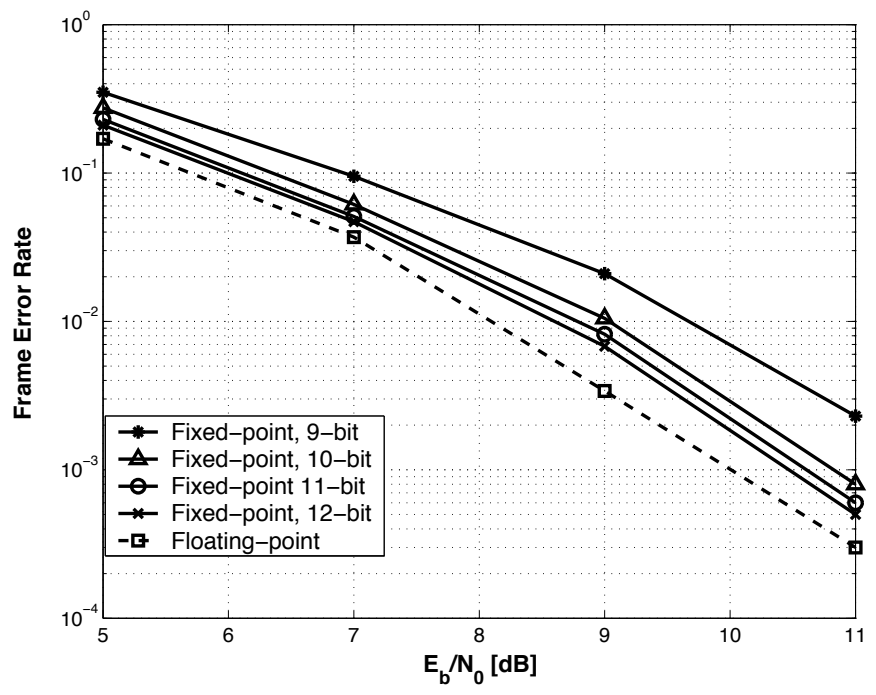

Fig. 11. Fixed vs. floating-point frame error rate performance of $\operatorname{BSSD}(16,100,100)$ detection; 4x4 16-QAM, Winner A1-NLOS channel. 10bit precision is chosen for implementation of BSSD algorithm and LDPC decoding.

\section{ASIC synthesis results}

The VHDL code for the bounded soft sphere detector that supports a system with four transmit/receive antennas with 16-QAM modulation is synthesized using the Synopsys front-end design compiler [15] and the IBM $90 \mathrm{~nm}$ CMOS technology. The total area cost of four search units is approximately $0.52 \mathrm{~mm}^{2}$, and the total search memory area size is $0.05 \mathrm{~mm}^{2}$, which corresponds to the total core area size of one BSSD detector of approximately $0.57 \mathrm{~mm}^{2}$. The total dynamic power dissipation for the clock frequency of $200 \mathrm{MHz}$ is approximately $43.45 \mathrm{~mW}$.

Table II shows detection throughput, area cost and frameerror rate performance of the proposed bounded soft sphere 


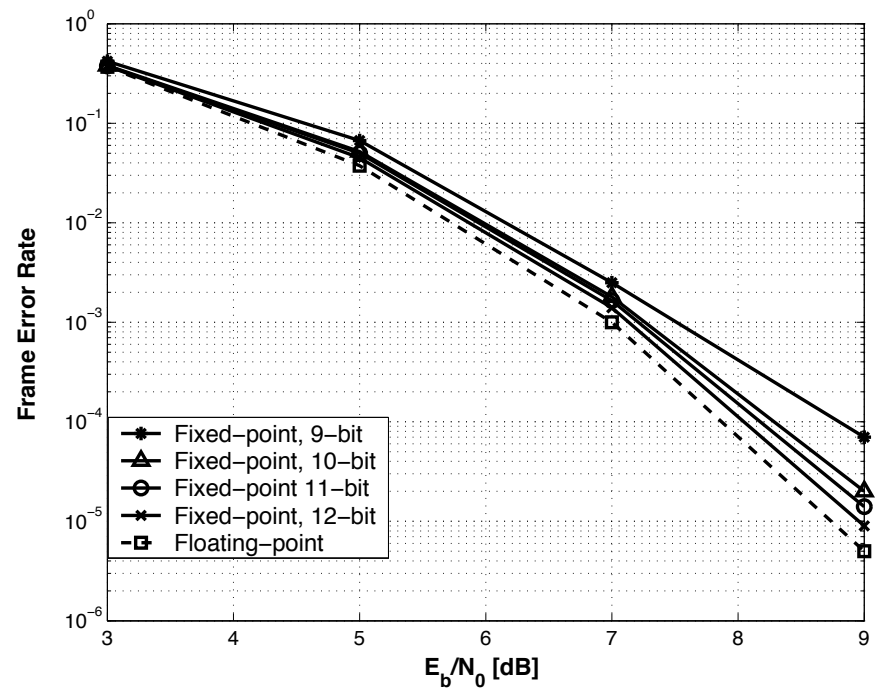

Fig. 12. Fixed vs. floating-point frame error rate performance of $\operatorname{BSSD}(16,100,100)$ detection; 4x4 16-QAM, Winner B1-NLOS channel. 10bit precision is used for BSSD algorithm and LDPC decoding.

TABLE I

DESIGN STATISTICS FOR THE SOFT SPHERE DETECTOR WITH BOUNDED SEARCH - BSSD $(16,100,100)$ ON VIRTEX2P70 FPGA.

\begin{tabular}{|c|c|c|}
\hline Resource & Utilized & Percentage \\
\hline \hline Slices & 5747 & $17 \%$ \\
\hline Flip-Flops & 3415 & $5 \%$ \\
\hline LUTs & 10662 & $16 \%$ \\
\hline Block RAMs & 23 & $7 \%$ \\
\hline
\end{tabular}

detector with pipelined search levels versus certain hardware implementations from the literature: the depth-first soft sphere detector from [6] with up to 256 search operations, the breadth-first soft K-best detectors from [8] and [16]. Our solution is significantly faster and has slightly better detection accuracy than the depth-first detector. On the other hand, although slower, the proposed BSSD detector has substantially better error-rate performance than the soft K-best detectors from [8], [16].

It is also important to note that the proposed bounded soft sphere detection (BSSD) algorithm has more potential for parallelization than the soft K-best approach. In both cases the pipelining of candidate-search in multiple search levels is assumed. If the number of available search units per search level is smaller than or equal to $K$, the processing throughput is decreased approximately $K$ times in both detectors. However, if the number of parallel search units is equal to $P$ per search level where $P$ is greater than $K$, then the throughput of the soft $\mathrm{K}$-best detector remains the same because there are no more than $K$ candidates per search level to be evaluated. Therefore, $P-K$ search units remain unutilized. On the other hand, all $P$ search units are uniformly utilized within the BSSD detector as long as there are at least $P$ preserved candidates in each search level.
TABLE II

COMPARISON OF SOFT SPHERE DETECTORS, $F_{C L K}=200 \mathrm{MHZ}, 0.13 \mu \mathrm{m}$ CMOS TECHNOLOGY, 4 TRANSMIT/RECEIVE ANTENNAS WITH 16-QAM.

\begin{tabular}{|c|c|c|c|c|}
\hline & {$[6]$} & {$[8]$} & {$[16]$} & BSSD \\
\hline \hline Av. Thr.[Mbps] & 7.68 & 106 & 320 & $\approx 72$ \\
\hline Area[ $\left.\mathrm{mm}^{2}\right]$ & 0.88 & 0.56 & 0.72 & $\approx 0.57$ \\
\hline SNR[dB] @ FER $=10^{-3}$ & 9.5 & 11 & 16 & 9.4 \\
\hline
\end{tabular}

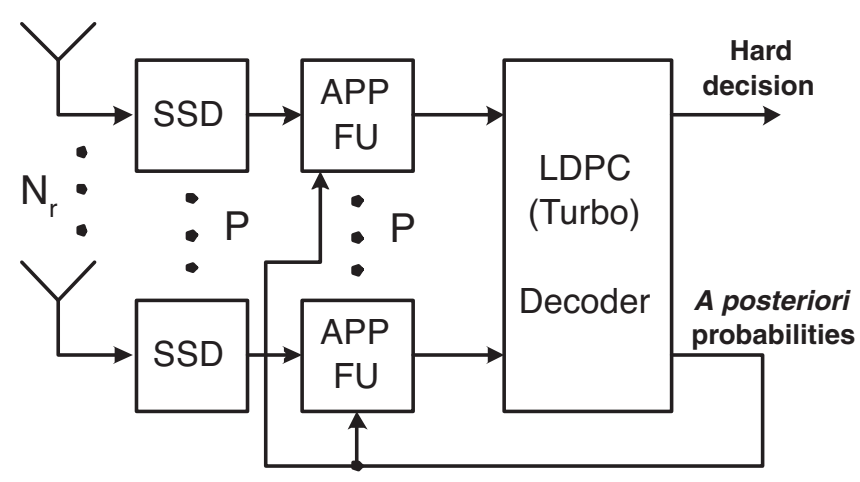

Fig. 13. Iterative receiver with parallel soft sphere detectors.

\section{ITERATIVE RECEIVERS FOR EMERGING WIRELESS STANDARDS: HARDWARE REQUIREMENTS FOR DOWNLINK RECEIVER TARGETING 1 GBPS DATA THROUGHPUT}

Wireless receivers for the next decade will target data-rates in the order of $1 \mathrm{GBps}$ for supporting various multimedia applications while providing excellent quality of service. Our goal in this work is also to estimate an area cost and power dissipation of a receiver physical layer (PHY) achieving such a high data throughput. In order to support data-rate specifications of emerging wireless standards, such as the $1 \mathrm{Gbps}$ downlink transmission, parallel soft sphere detectors (SSDs) need to be utilized at the receiver PHY. Parallel detectors are interfaced with an outer soft-input soft-output decoder, such as the LDPC or Turbo decoder, as illustrated in Fig. 13. It is again assumed a wireless system with four transmit/receive antennas, and 16-QAM modulation.

Although the emerging 3GPP-LTE standard specifies Turbo decoding at the receiver, the LDPC decoding is also an excellent candidate for outer decoding. High-speed flexible LDPC decoder architectures are well-known in the art [17], [18], [19], [20], and the principle for estimating a latency of iterative receiver with soft sphere detection is independent of decoding scheme. The codeword size for the outer LDPC decoder is assumed to be 1944 bits which is one particular specification for the emerging IEEE 802.11n standard [21], the code rate is $1 / 2$, maximum number of inner iterations of layered belief propagation algorithm is 15 . The area size of the flexible LDPC decoder implemented in [22] is approximately $3.3 \mathrm{~mm}^{2}$ if the Chartered $0.13 \mu \mathrm{m}$ CMOS technology is used for the ASIC synthesis. The implemented decoder supports several codeword sizes up to 1944 bits and multiple code rates between $1 / 2$ and $5 / 6$. The decoding latency is approximately $4.95 \mu \mathrm{sec}$ for the clock frequency of $200 \mathrm{MHz}$. There are up to four outer iterations between inner soft sphere detector and LDPC decoder. As illustrated in Fig. 13, the outer LDPC 


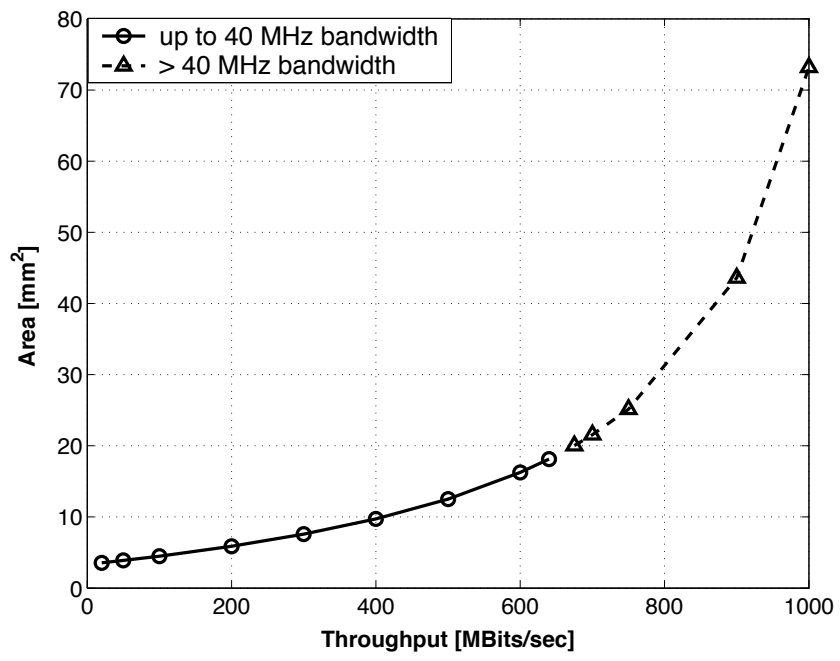

Fig. 14. Area cost versus achievable data-rates; downlink receiver with $\operatorname{BSSD}(16,100,100)$, LDPC decoder, and two outer iterations.

decoder is interfaced with APP units of parallel SSDs, and not directly with SSDs.

Bounded soft sphere detection $\operatorname{BSSD}(16,100,100)$ proposed in Section III-A is utilized for parallel detection in Fig. 13. The candidate-search at four different search levels (transmit antennas) can be performed simultaneously. Assuming the typical clock frequency for the ASIC design of $200 \mathrm{MHz}$, and the latency of one search operation of about two clock cycles, the pipelined data throughput is approximately $72 \mathrm{Mbps}$ per soft sphere detector. As it is presented in Section $\mathrm{V}-\mathrm{C}$, the area size of a single soft sphere detector (including the search memory and the APP function unit) is about $0.57 \mathrm{~mm}^{2}$.

The latency of the APP function unit and the number of final candidates affects the latency of outer feedback from decoder to detector. There are approximately 25 final candidates in the case of Rayleigh fading sub-carrier channels for our pre-determined value of sphere radius; the number of final candidates for indoor Winner A1-NLOS and outdoor Winner B1-NLOS channels is smaller - about eight for both environments. The latency of updating reliability messages based on a single final vector-candidate is approximately one clock cycle, assuming a clock frequency of $200 \mathrm{MHz}$. Therefore, the total latency for updating the a posteriori messages based on the entire list of candidates is approximately $0.125 \mu \mathrm{sec}$. The full latency of the iterative detection-decoding process can be determined as in (15), where $C_{S}$ is the decoder codeword size in bits (1944 bits in this particular case), $P$ is the number of soft sphere detectors from Fig. 13 that operates in parallel, Search_lat. represents the latency of the candidate-search process (either pipelined or non-pipelined implementation), $A P P \_l a t$. is the latency of the APP function unit, Dec_lat. represents the latency of outer decoder, and Iter is the maximum number of outer iterations between decoder and soft sphere detector (i.e., APP function unit).

The data throughput $T h r$ at the receiver is determined as:

$$
T h r=C_{S} / I D D \_l a t .
$$

The area cost of iterative detection-decoding part of the receiver is equal to summation of areas of parallel soft sphere

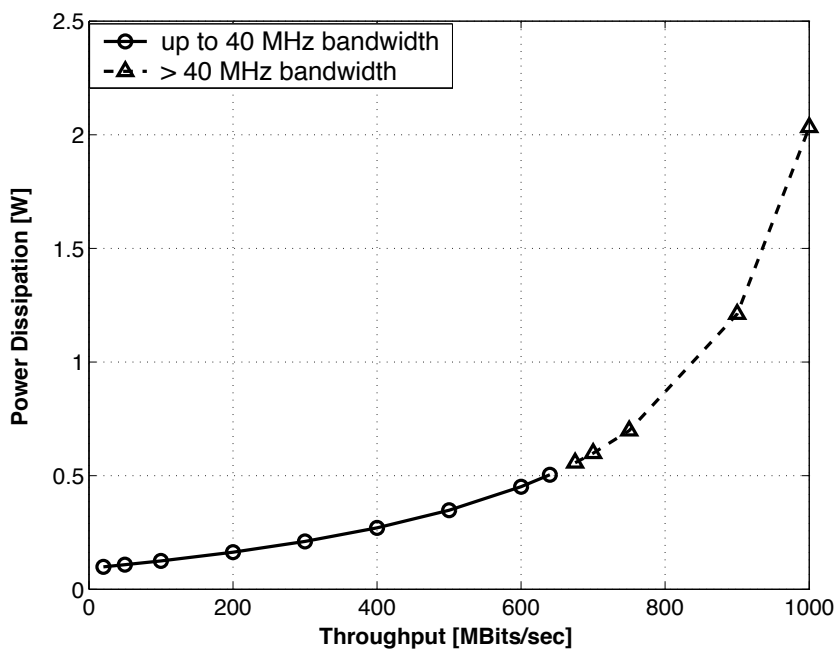

Fig. 15. Power dissipation versus achievable data-rates; downlink receiver with $\operatorname{BSSD}(16,100,100)$, LDPC decoder, and two outer iterations.

detectors and the area of outer decoder:

$$
\text { Area }=P \cdot S S D \_a r e a+D e c \_a r e a,
$$

where SSD_area is the area of one soft sphere detector $\left(0.57 \mathrm{~mm}^{2}\right)$, and Dec_area is the area of the LDPC decoder (3.3 $\left.\mathrm{mm}^{2}\right)$. After combining (15), (16), and (17), we derive a relation between the area cost of the iterative detectiondecoding part of the receiver and desired data throughput $T h r$ as in (18).

Assuming a typical power dissipation for the $0.13 \mu \mathrm{m}$ CMOS technology of $10 \mathrm{nW} / \mathrm{MHz} /$ gate [23], it can be also determined a relation between desired data throughput and power dissipation of the iterative detection-decoding part of receiver illustrated in Fig. 13. Figure 14 and Fig. 15 show the area-cost and power dissipation of the detection-decoding part of the receiver as a function of data throughput, if the 16-QAM modulation is applied at a transmitter. If the targeted data throughput is $1 \mathrm{Gbps}$ and two outer iterations between bounded soft sphere detector and LDPC decoder are assumed, then the estimated area cost of the iterative detection-decoding part of the receiver is about $73 \mathrm{~mm}^{2}$ and power dissipation is $2.05 \mathrm{~W}$, for clock frequency of $200 \mathrm{MHz}$. By knowing the total area cost of the iterative detection-decoding part of the receiver (i.e., $73 \mathrm{~mm}^{2}$ ), the area of outer LDPC decoder (i.e., $3.3 \mathrm{~mm}^{2}$ ) and the area of one SSD including the APP function unit (i.e., $0.57 \mathrm{~mm}^{2}$ ), it can be determined from (17) that there are $P=123$ soft sphere detectors and APP function units in Fig. 13 that operate in parallel.

It can be observed from Fig. 14 and Fig. 15 that in order to achieve high data throughput at the receiver and assuming four transmit/receive antennas with 16-QAM modulation, the channel bandwidth above $40 \mathrm{MHz}$ is required, which represents a typical upper bound for the bandwidth of today's wireless systems and standards. For example, for the data throughput of $1 \mathrm{Gbps}$ and 16-QAM modulation, the channel bandwidth of $62.5 \mathrm{MHz}$ is needed.

In the case of a system with four transmit/receive antennas and 64-QAM modulation, the channel bandwidth of $40 \mathrm{MHz}$ is sufficient to provide data throughput of 1 Gbps at the 


$$
\begin{aligned}
& \text { IDD_lat. }=\frac{C_{S}}{P \cdot\left(N_{t} \cdot M_{C}\right)}\left(\text { Search_lat. }+ \text { Iter } \cdot A P P \_l a t .\right)+I t e r \cdot D e c \_l a t ., \\
& \text { Area }=\frac{C_{S}(\text { Search_lat }+ \text { Iter } \cdot \text { APP_lat }) \cdot \mathbf{T h r}}{\left(N_{t} \cdot M_{C}\right) \cdot\left(C_{S}-\mathbf{T h r} \cdot \text { Iter } \cdot \text { Dec_lat }\right)} \cdot S S D \_a r e a+D e c \_a r e a .
\end{aligned}
$$

receiver side. The required area cost of the iterative detectiondecoding part of the receiver is however larger: $155 \mathrm{~mm}^{2}$, which corresponds to a power dissipation of about $4.25 \mathrm{~W}$ for $0.13 \mu \mathrm{m}$ CMOS technology and clock frequency of $200 \mathrm{MHz}$. The estimated power dissipation is however more than twice smaller if the latest $45 \mathrm{~nm}$ CMOS technology is utilized. Larger power dissipation and area size than in the case of 16QAM modulation is due to a larger implementation complexity of soft sphere decoder since there are more candidates to be evaluated. Also, memory size for storage of candidates during the search process is larger than in the case of 16QAM modulation.

\section{SUMmARY AND CONCLUSIONS}

The bounded soft sphere detection (BSSD) is proposed with probabilistically determined and variable search bounds per search level (i.e., per transmit antenna) as the inner detection part of iterative wireless receiver. The proposed BSSD algorithm represents efficient solution in terms of computational complexity and error-rate performance. Excellent error-rate performance is achieved thanks to better utilization of candidates in every search level providing better reliability information for outer decoder. The error-rate performance of the BSSD algorithm is superior compared to the soft Kbest approach [8] also considered as a possible solution for future downlink mobile receivers, as well as compared to the soft MMSE equalization with outer feedback from soft decoder [24], [25] that is currently implemented in practical systems. The proposed detection approach can achieve data throughput in the order of $1 \mathrm{Gbps}$ as the target data-rate for emerging and future wireless systems with affordable implementation cost and power dissipation. At the same time, high spectral efficiency is provided since the wireless system with four transmit antennas and 16-QAM constellation size is supported.

The proposed hardware solution for the BSSD detector is competitive in its speed, detection accuracy and area cost with the state of the art solutions. It improves by more than five times data throughput of the depth-first sphere detector [6], while the implementation complexity (area cost) and detection accuracy are comparable. It also improves upon the detection accuracy of the soft K-best high-speed implementations [8], [26], while the data throughput is comparable when pipelining of the candidate-search for multiple transmit antennas is applied. Finally, it is shown that the downlink data rate requirements of emerging wireless standards can be achieved. For example, 1 Gbps downlink data throughput is achieved with area cost of the iterative detector-decoder of approximately $73 \mathrm{~mm}^{2}$ and with power dissipation of about $2 \mathrm{~W}$, if $0.13 \mu \mathrm{m}$ CMOS technology is utilized.

\section{ACKNOWLEDGMENT}

This work was supported in part by Nokia Corporation and by NSF under grants CCF-0541363, CNS-0619767, CNS0551692 , CNS-0321266.

\section{REFERENCES}

[1] G.J. Foschini, "Layered space-time architecture for wireless communication in a fading environment when using multi-element antennas," Bell Labs. Tech. J., vol. 1, pp. 41-59, 1996.

[2] E. Casas and C. Leung, "OFDM for data communication over mobile radio FM channels. I. Analysis and experimental results," IEEE Trans. Commun., vol. 39, pp. 783-793, May 1991.

[3] B. Lu, Guosen Yue, and Xiaodong Wang, "Performance analysis and design optimization of LDPC-coded MIMO OFDM systems," IEEE Transactions on Signal Processing [see also IEEE Trans. Acoustics, Speech, Signal Processing], vol. 52, pp. 348-361, Feb. 2004.

[4] D. Wang, J. Hua, X. Gao, Y. X., M. Weckerle, and E. Costa, "Turbo detection and decoding for single-carrier block transmission systems," in 15th IEEE International Symposium on Personal Indoor and Mobile Radio Communications (PIMRC 2004), pp. 1163-1167, Sept. 2004.

[5] B.M. Hochwald and S. ten Brink, "Achieving near-capacity on a multiple-antenna channel," IEEE Trans. Commun., vol. 51, pp. 389-399, March 2003.

[6] D. Garrett, L. Davis, S. ten Brink, B. Hochwald, and G. Knagge, "Silicon complexity for maximum likelihood MIMO detection using spherical decoding," IEEE J. Solid-State Circuits, vol. 39, pp. 1544-1552, Sept. 2004.

[7] Y. Dai, S. Sun, and Z. Lei, “A comparative study of QRD-M detection and sphere decoding for MIMO-OFDM systems," in 16th IEEE International Symposium on Personal Indoor and Mobile Radio Communications (PIMRC 2005), pp. 186-190, Sept. 2005.

[8] Z. Guo and P. Nilsson, "Algorithm and implementation of the K-best sphere decoding for MIMO detection," IEEE J. Select. Areas Commun., vol. 24, pp. 491-503, March 2006.

[9] 3rd Generation Partnership Project (3GPP), technical specification group radio access network, physical layer aspects for evolved UTRA. Technical report, Release 7, November 2005.

[10] U. Fincke and M. Pohst, "Improved methods for calculating vectors of short length in a lattice, including a complexity analysis," Math. Comput., vol. 44, pp. 463-471, Apr. 1985.

[11] Z. Guo and P. Nilsson, "VLSI implementation issues of lattice decoders for MIMO systems," in ISCAS '04. Proc. 2004 International Symposium on Circuits and Systems, pp. 477-480, May 2004.

[12] C.P. Schnorr and M. Euchner, "Lattice basis raduction: Improved practical algorithms and solving subset sum problems," Math. Programming, vol. 66, pp. 181-191, 1994.

[13] Y. Dai, S. Sun, and Z. Lei, "Performance comparison between coded V-BLAST and GSTTC MIMO-OFDM systems," in PIMRC 2004. 15th IEEE International Symposium on Personal, Indoor and Mobile Radio Communications, pp. 2581-2585, Sept. 2004.

[14] J. Salo, G. Del Galdo, J. Salmi, P. Kysti, M. Milojevic, D. Laselva, and C. Schneider, "MATLAB implementation of the 3GPP Spatial Channel Model (3GPP TR 25.996).” On-line, Jan. 2005. http://www.tkk.fi/Units/Radio/scm/.

[15] "Synopsys products: RTL synthesis." http://www.synopsys.com/products/logic/design_compiler.htm.

[16] M. Wenk, A. Burg, M. Zellweger, C. Studer, and W. Fichtner, "VLSI implementation of the list sphere algorithm," in 24th Norchip Conference, pp. 107-110, Nov. 2006.

[17] M.M. Mansour and N.R. Shanbhag, "High-throughput LDPC decoders," IEEE Trans. VLSI Syst., vol. 11, pp. 976-996, Dec. 2003. 
[18] T. Bhatt, V. Sundaramurthy, V. Stolpman, and D. McCain, "Pipelined block-serial decoder architecture for structured LDPC codes," in IEEE International Conference on Acoustics, Speech and Signal Processing (ICASSP), pp. IV-225 - IV-228, May 2006.

[19] P. Radosavljevic, A. de Baynast, M. Karkooti, and J.R. Cavallaro, "Multi-rate high-throughput LDPC decoder: tradeoff analysis between decoding throughput and area," in 17th IEEE International Symposium on Personal, Indoor and Mobile Radio Communications (PIMRC), Sept. 2006.

[20] M. Karkooti, P. Radosavljevic, and J.R. Cavallaro, "Configurable high throughput irregular LDPC decoder architecture: tradeoff analysis and implementation," in IEEE 17th International Conference on Applicationspecific Systems, Architectures and Processors (ASAP), pp. 360-367, Sept. 2006.

[21] IEEE 802.11 Wireless LANs WWiSE Proposal: High Throughput extension to the 802.11 Standard. IEEE 11-04-0886-00-000n.

[22] P. Radosavljevic, M. Karkooti, A. de Baynast, and J.R. Cavallaro, "Tradeoff analysis and architecture design of high throughput irregular LDPC decoders." submitted to IEEE Trans. Circuits Syst. I: Regular Papers, Nov. 2006.

[23] "0.13-micron CMOS ASIC TC280 Family." http://www.toshiba.com/taec/components/Generic/45115c0107.pdf.

[24] M. Tuchler, R. Koetter, and A.C. Singer, "Turbo equalization: principles and new results," IEEE Trans. Commun., vol. 50, pp. 754-767, May 2002.

[25] M. Tuchler, A.C. Singer, and R. Koetter, "Minimum mean squared error equalization using a priori information," IEEE Transactions on Signal Processing [see also IEEE Trans. Acoustics, Speech, Signal Processing], vol. 50, pp. 673-683, March 2002.

[26] A. Burg, M. Borgmann, M. Wenk, M. Zellweger, W. Fichtner, and H. Bolcskei, "VLSI implementation of MIMO detection using the sphere decoding algorithm," IEEE J. Solid-State Circuits, vol. 40, pp. 15661577, July 2005.

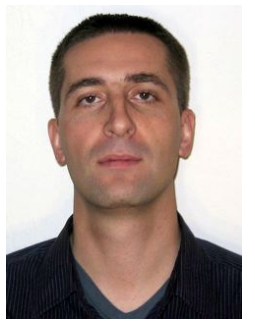

Predrag Radosavljevic received the B.S. degree in electrical engineering from University of Belgrade, Serbia in 2000, the M.S. degree in 2004 and the Ph.D. degree in 2008, all in electrical engineering from the Department of electrical and computer engineering, Rice University, Houston, Texas. His research interest includes design of detection/decoding algorithms and architectures for wireless communication systems. He is currently a senior technical advisor with Patterson\&Sheridan, LLP. He is practicing preparation and prosecution of patent applications with emphasis on wireless communications, electronics, and signal processing.

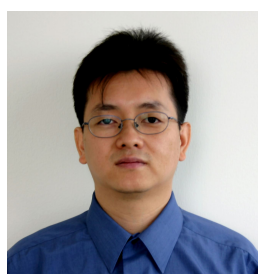

Yuanbin Guo graduated with a Ph.D. degree in Electrical and Computer Engineering from Rice University in Houston, Texas. He is currently a Senior MTS (Technical Lead) at Huawei. He was a Research Specialist with the renewed Nokia Siemens Networks from 2007-2008, and a (Senior) Research Engineer with Nokia Research Center, then Nokia Networks from 2002-2007. He also worked for BellLabs Lucent in the Intelligent Network from 19992000. He obtained his MS from Beijing University of Posts and Telecommunications in 1999 and BS from Peking University in 1996, both from Electrical and Electronics Engineering. His research interests are in the areas of wireless communications, VLSI implementation and rapid prototyping of wireless systems, including CDMA, OFDM related standards such as WCDMA/HSDPA, WiMax and LTE.

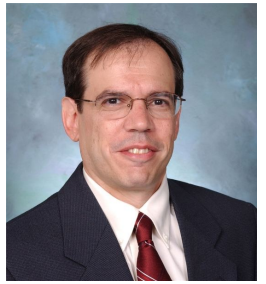

Joseph R. Cavallaro received the B.S. degree from the University of Pennsylvania, Philadelphia, $\mathrm{Pa}$, in 1981, the M.S. degree from Princeton University, Princeton, NJ, in 1982, and the Ph.D. degree from Cornell University, Ithaca, NY, in 1988, all in electrical engineering. From 1981 to 1983, he was with AT\&T Bell Laboratories, Holmdel, NJ. In 1988, he joined the faculty of Rice University, Houston, Texas, where he is currently a Professor of electrical and computer engineering. His research interests include computer arithmetic, VLSI design and micro-lithography, and DSP and VLSI architectures for applications in wireless communications. 